\title{
A Study of Verbal Humor in Sitcom The Big Bang Theory from the Perspective of Memetics
}

\author{
Xiaoli Bao ${ }^{1}$ \\ ${ }^{1}$ School of Foreign Language, Inner Mongolia University for Nationalities, Tongliao, Inner Mongolia, China \\ Correspondence: Xiaoli Bao, School of Foreign Language, Inner Mongolia University for Nationalities, Tongliao, \\ Inner Mongolia, China. E-mail: nmbaoxiaoli@163.com
}

Received: February 6, 2017 Accepted: February 19, 2017 Online Published: February 24, 2017

doi:10.5539/ells.v7n1p86 URL: http://doi.org/10.5539/ells.v7n1p86

\begin{abstract}
Verbal humor has always been in hot discussion in the academic field recently. The Big Bang Theory is a popular American sitcoms. The show is primarily centered on five characters living in Pasadena, California. The greekiness and intellect of the four men are contrasted for comic effect with Penny's social skills and common sense. On the basis of the previous study, this thesis tries to analyze verbal humor in sitcom The Big Bang Theory from the perspective of Memetics. Firstly, the article introduces the general definition and features of verbal humor, and gives us a general overview of the memetic theory. Secondly, the article concentrates on the causes of humor generation and the process of humor generation from perspective of memetics. Lastly, the author hopes this article can help us to appreciate verbal humor in English sitcoms.
\end{abstract}

Keywords: memetics, language meme, verbal humor, study

\section{Introduction}

\subsection{Background of the Research}

Memetics is a new theory which is based on the Dawkins' theory of evolution to interpret cultural evolution (Dawkins, 1976). It interprets the common relationship among things and the evolutionary law of the spreading culture - the essential characteristics, from the diachronic and synchronic points. The core in memetics is the term "meme" which was firstly coined by the British evolutionary biologist Richard Dawkins in his book The Selfish Gene in 1976. Dawkins Richard coined the cultural evolutionary unit "meme" based on exploring gene's self-replication and competition to speed up biological evolution in The Selfish Gene. And then foreign scholars in various study fields gradually began to show more interests in it and have made a lot of achievements in their own fields. In 1999, memetics was introduced into China. From then on, many Chinese scholars have devoted to studying it and applying it to wider scopes. The language meme is one of manifestation of memetic application to linguistics. However, studies of the language meme are few, and of verbal humor from language meme are even fewer.

\subsection{Motivation and Significance of the Study}

First, the thesis is of great value to further the exploration of verbal humor from the perspective of language meme. Specifically speaking, compared with the study of humor from the perspective of pragmatics like cooperative principle, polite principle, speech act theory, conceptual blending theory etc, language meme can interpret verbal humor in a more understandable way. Although few essays indeed explain verbal humor from perspective of language meme, they just list the modes of language meme in verbal humor but don't explain how humorous effect engenders.

Second, situation comedies The Bigbang Theory are so popular there must be some reasons behind it. If the reasons can be revealed clearly through the study of verbal humors in a memetic angle, then it is helpful for television and film makers to produce more meaningful and educational television series and films, in the long run, it can not only help individuals but also can help to build a more harmonious and healthier society.

Third, the thesis has significant practical importance to English learning and teaching. As we have known the generation process of verbal humor, it will become much easier for us to appreciate verbal humor in English sitcoms which is beneficial for us to improve English 


\subsection{The Content of Study}

Big Bang Theory which is famous for its ubiquitous humor mainly tells about four physics genius who are good friends. Their special behavior and the witty dialogue during the daily life have brought the audience great laughter and happiness. And the verbal humor of the sitcom is the main focus of the thesis. This thesis mainly concentrates on the process of the generation and transmission of verbal humor from the perspective of memetics. What we will discuss is the causes of humor generation and the process of humor generation.

\section{Literature Review}

The following section mainly presents the theoretical frame of the thesis.

\subsection{Humor}

\subsubsection{Definition, Classification and Feature of Humor}

Humor means the faculty of recognizing something that is comical or laughable when one sees, hears it, sometimes inducing you to smile or laugh, or the quality of something which arouses such amusement (Cambridge Dictionary of American English, 2000, p. 426). Humor is the ability of discovering comic factors in life and the ability of creating and reflecting them in art; an artistic means with major aesthetic features of relaxed, bantering and meaningful laughter (Word Ocean, 1999, p. 2236).

Humor can be divided into different types according to different criteria and different scholars. Lin Yutang (2000) divides humor into high humor and low humor, the former refers to elegant and noble humors, while the latter refers to popular humors used by daily life. Tan Daren divides humor into communicative, ironic, pure and black humors according to the functions and purposes. (1997, p. 72) Humor can also be divided into joke, irony, satire and so forth according to the expressing styles, while, according to the contents, humor can be divided into religious, sexual, political humors.

In order to understand how humor works, the researchers, from different linguistics perspectives, concludes the features of humor. The incongruity theory implies that it seems that verbal humor is inconsistent and inharmonious. $\mathrm{Xu}$ Lixin believes that pleasure; laughter, unexpectedness, playful spirit and witty spirit are all characteristics of humor (2003, p. 20). The Bigbang Theory fully shows the features of humor.

\subsection{Meme and Memetics}

2.2.1 Origin, Definitions, Classifications of Meme, and Features of Successful Meme, Stages of Meme Replication

Richard Dawkins put forward "meme" in his book The Selfish Gene. He mainly discussed on how gene promotes the evolution of the living world through self- replication and competition. Blackmore, one of Dawkins' students, that developed and enriched Dawkins' study on meme. Blackmore made memetics and independent branch of linguistic study. She pointed out in his book The Meme Machine (1999) that just like genes try very hard to enter the body of next generation selfishly, meme is the same, they compete with each other to enter the brain of next host or any other tools like books, computers. Gene formed the world pattern and structure; it is meme that finally formed the pattern of culture and psychological pattern. She also pointed that, it is meme that made the evolution of concept, and the evolution must be beneficial for the self-replication of meme not for the self-replication of gene. That is a huge contribution of her to the study of memetics.

In 2005, the term meme entered The Oxford Dictionary of English. Oxford Dictionary of English defines meme as bellow: Meme: an element of a culture or system of behavior that may be considered to be passed from one individual to another by non-genetic means, esp. imitation. Derivatives: memetic a., memetics $n$. the science of memes. memeplex n. abbreviated from "co-adapted meme complex"; a group of memes passed on together, e.g. religions, political ideologies and belief systems. meme pool $\mathrm{n}$. all the memes in a population at a particular time (Soanes \& Stevenson, 2005, p. 952). Blackmore broadened the concept of meme in a large sense. She believes that any information that can be imitated and transmitted can be called meme. She also claims that imitation is the way of the replication of meme. Thus, if imitation occurs, and something is transmitted, then the thing being transmitted is called meme. There are other definitions of meme, it is difficult to draw a clear and precise definition of it.

Scholars divide many types of memes according to different criteria. There are three types of meme stated in this thesis. The first kind is meme complexes and singular memes, the second kind is beneficial memes and harmful memes, the last kind if powerful memes and weak memes.

In order to replicate and transmit successfully, memes should have certain characteristics. Dawkins listed three characteristics in this book titled The Selfish Gene. He thinks "imitation is how memes can replicate. But just as 
not all genes that can replicate do so successfully, so some memes are more successful in the meme pool than others.Butin general, they must be the same as those discussed: longevity, fecundity and copy-fidelity" (Dawkins, 1976, p. 240).

Heylighen suggested that the transmission of a successful meme has a four-stage life cycle, assimilation, retention, expression and transmission (1998, p. 27). The first stage of meme replication is assimilation. In order to enter into the mind of a new host, a new meme has to try its best to attract the attention from the host. Only when a meme is noticed and comprehended by a new host can it be accepted by a host who first encounter it. The second stage of the meme replication is retention. After been noticed and understood by a new host, the new meme has to try every means to stay in the mind of the new host. The longer a new meme can stay in the mind of a new host, the more likely the chance of being remembered and transmitted is. The third stage of meme replication is expression. Expression means how a new meme is transmitted, by what forms, such as language, text, picture, behavior or other forms and so on. The last stage of the replication of a meme is transmission. In this stage, a new meme can be transmitted to a new host by various vectors, such as books, films, hardware and other communication media. The transmission of a meme must go through the above four stages, it also has to be mentioned that in each stage there are selections, only the most powerful memes can stay, for these most powerful memes possess all the characteristics a successful meme has, longevity, fecundity and copying-fidelity. Those weak memes might be wiped out in any stage of the four stages. In our language, only these most powerful memes can survive and be remembered by people after four stages of its replication.

\subsubsection{Language Meme}

Language and meme are connected with each other closely. On the one hand, language is a medium to transmit memes, on the other hand, as gene of culture, meme exert dramatic influence on the existence and development of language and culture.

\subsubsection{Replication and Transmission of Language Meme}

Language is one of the most important media to spread meme. In 2001, Blackmore also writes like that "the ability of human language provides meme but not gene with superiority in selection. Once a meme appears, it will change the circumstance in which genes are selected, and consequently forces genes to evolve better for the transmission of memes. In the other words, the function of language is to transmit memes." (Blackmore, 2001, p. 173). Memes can also influence the existence and development of language. In the book of The Meme Machine, Blackmore says "any information that can be copied through imitation can be called meme" (1999, p. 66)

\subsubsection{Types of Language Memes}

Blackmore says in The Meme Machine "language meme consists of two types: memetic genotype and memetic phenotype." (1999, p. 193).

Memetic genotype is a type of memes that may be presented directly or with various forms, while the content of which is remained unchangeable. It can be divided into two subcategories, the first subcategory is the same information transmitted directly, idioms, proverbs, sayings, poems, famous public speeches and even some paragraphs are all fall into this type. For example, people will use the saying "no pains, no gains" to emphasis the importance of hardworking. While, when someone use the phrase likes "that's one small step for a man, one giant leap for mankind", he may want to use this sentence to show that he made something meaningful and significant. The second subcategory is the same information transmitted in varied forms, such as a teacher can be called "Confucian scholar", "master", "Mr.", "teacher", "gardener" and so on. Even though, the titles are different, the content is the same, all the titles refers to someone who teach knowledge, skills and so on.

Memetic phenotype is the identical set of pattern being followed by different contents. This type of memes can be divided into three subcategories. The first subcategory is homophone, such as an advertisement "good-buy" it is the homophone of good-bye, advertisers use this homophone to attract the buyer to their products which is a real bargain. "More sun and air for your son and heir" is another example. The second subcategory of phenotype memes is homograph, that is to say, different associative meaning are expressed by the same form, such as, when someone say "you looks cloudy" he may mean that the man he refers is sad and depressed. Cloudy is an adjective used for describing the weather, when it is used to describe someone, it arouses the association of whether. The third subcategory of phenotype memes is structural homonymy. "If winter comes, can spring be far behind?" is a very famous sentence written by Percy Bysshe Shelley, after the appearance of it, it has been change for many times, such as "if summer holiday comes, can winter holiday be far behind?", "if your mother comes, can your father be far behind?", "if failure comes, can success be far behind?" and so forth. 


\subsubsection{Meme Studies at Home and Abroad}

Since language memes is so important in the existence and development of language that the applications of language meme also attracted a lot of attentions from many fields. In 1997, Deacon published a book called The Symbolic Species, Lynch put forward a book entitled Thought Contagion in 1996, The Meme Machine published by Blackmore in 1999. There are also some scholars who paid their attention to the study of memetics. In 2007, He Ziran published a book entitled New Developments in Pragmatics: Relevance, Adaptation and Memetics which shed new light on the study of memetic field. Chesterman (1997) is the first man linked memetics with translation study. As language memes are selections of culture, the study of culture is closely related with language memes. Through the study of language memes, the origin, development, existence, appearance, disappearance of some language and culture can be explained clearly. Since language memes are selections of successful memes, language meme can also be applied to language teaching, such as, reciting, writing, speaking, even listening study.

\section{Causes of Generation of Verbal Humor from the Bigbang Theory}

\subsection{Meme Variation}

Meme variations refer to any change happens in the process of replication and transmission of memes which mainly include errors happens in the replication, recombined memeplexes, modification of singular memes. According to He Ziran, "meme can vary in form or content in the process of replication and transmission. (2009, p. 341) Meme variation is the combination of a language meme and new component, it usually contains two parts, one part is language meme which is already known to people, another part is meme variant which is unknown to people. That is to say, meme variation is a combination of both novelty and familiarity. In order to understand and appreciate meme variations, cognitive transformation from familiar component to novel component is required. Koestler (1964) says "in order to produce surprising results of humor, people who have sense of humor should have a little bit of creativity, and the ability to break the conventional thinking way." Thus, either old forms filled with new contents or old contents expressed with new forms can make get a feeling of both novelty and familiarity of meme variations in verbal humors.

From the above opinions, it can be seen meme can promote the transmission and development of language. Language is the main carrier of meme. Meme variation can affect the generation of verbal humor. Let's take the following conversation as an example.

Sheldon: Scissors cuts paper, paper covers rock, rock crushes lizard, lizard poisons Spock, Spock smashes scissors, scissors decapitates lizard, lizard eats paper, paper disproves Spock, Spock vaporizes rock, and as it always has, rock crushes scissors. (Episode 4, Season1)

The above one is the classic verbal humor from the The Bigbang Theory. What we usually play is the classic "Rock, Paper, Scissors" game. The game is known by the people all across the world and has been passed from one generation to another generation. Thus it is a successful meme. But when Sheldon plays the game, he creates a new version based on the classic. The classic version "Rock, Paper, Scissors" is transformed into "Rock, Paper, Scissors, Lizard, Spock". That is, the meme "Rock, Paper, Scissors" get meme variation during the replication and transmission of the meme, thus leading to the humorous effect.

\subsection{Disharmony between Default Context and New Context}

Default context refers to the context that is generally known and is highlighted in language meme. As we are surrounded by Chinese culture, it will be a little more difficult for us to have a quite good knowledge of the default context of English language meme. Thus it will be not easy for Chinese to understand an English humor. The disharmony between default context and new context of language meme can lead to the humorous effects. Thus even though the new context of language is provided, the lack of the knowledge of default context can also fail the understanding of the verbal humor. The language meme that is contained in verbal humor all have its own default context and new context. Language meme contained in verbal humor transformed from the default context into new context. The sudden perception of disharmony between default context and new context of language meme can generate humorous effects. Humorous effects can be gained through the breaking of the harmony between the contexts in the aspects of word or sentence or paragraph. The generation of verbal humor from the disharmony between contexts from the perspective of vocabulary or culture will be discussed. The examples will be taken from The Bigbang Theory.

First, the disharmony is elaborated from the perspective of vocabulary. For example: 


\section{Example 1}

(In the insect lab)

Howard: You're like a girl. They're just bugs.

Rajesh: Well, I don't like bugs, okay? They freak me out.

Sheldon: Interesting, you're afraid of insects and women, ladybugs must render you catatonic. (Episode 2, Season 1)

From the above example, we can see the appropriate use of the word "ladybugs" generates the humorous effects. The generation of humorous effect in "interesting, ...you catatonic" lies in the disharmony between the default context and the new context of the language meme "ladybugs". The default context of the language meme "ladybug" is very simple. The definition of ladybug is "small round bright-colored and spotted beetle that usually feeds on aphids and other insect pests". However, when it is use in the new context, it can generate humorous effect. In the lab, Rajesh is afraid of bugs. At the same time, he is afraid of talking with girls. Therefore, Sheldon use the word "ladybug" to tease him by saying that "you're afraid ...render you catatonic." The spelling of "ladybug" is the combination of "lady" and "bug". The disharmony between the default context and the new context causes the humorous effect. Jumping from the default context to the new context of "ladybug" lead to the incongruity which can be resolved in laughter in a harmonious way. The default context is known and the new context is unknown. The cognitive transformation which is caused by the leaping from the default context to the new context turns the expectation into nothing, which generates humorous effects. However, when the conversation is translated into Chinese, the humorous effect will be disappeared. Because the generation of the humorous effect in this conversation mainly depends on the new context of the spelling of the word "ladybug". When it is translated into Chinese, the new context of the English version will disappear. Thus the humorous effect will fail.

\section{Example 2}

Missy: I always bragging to my friends about my brother the rocket scientist.

Sheldon: You tell people I'm rocket scientist?

Missy: Well, yeah.

Sheldon: I'm a theoretical physicist.

Missy: What's the difference?

Sheldon: What's the difference?! My god! Why don't you just scientist them that I'm a toll-taker at the Golden Gate Bridge?! Rocket Scientist. How humiliating! (Episode 6, Season1)

In this conversation, the humor mainly lies in the sentence "What's the difference?! My god! Why don't you just tell them that I'm a toll-taker at the Golden Gate Bridge?! Rocket Scientist. How humiliating". It is the disharmony between the default context and the new context of the word "rocket scientist" that causes the humor. It is known to all that to be a rocket scientist is very hard since it requires someone to be extremely clever and talented. So Missy always brag to her friends that her brother is a rocket scientist. And Sheldon is just a theoretical physicist. However, Sheldon is angry at calling him rocket and rather to be a toll-taker at the Golden Gate Bridge. He thus says "Why don't you just tell them that I'm a toll-taker at the Golden Gate Bridge?! Rocket scientist. How humiliating!" The humorous effect is generated. In the default context, "rocket scientist" is usually used as a commendatory term while it is transformed to a derogatory term in the new context. The disharmony between the two contexts lead to the generation of humorous effect.

Second, he disharmony is elaborated from the perspective of culture. For example:

Example 3

Leonard: If we do get a new friend, he should be a guy you can trust. You know, a guy who has your back.

Howard: And he should have a lot of money and live in a cool place down by the beach where we could throw parties.

Sheldon: And he should share our love of technology.

Howard: And he should know a lot of women.

Leonard: Okay, let's see: money, women, technology. Okay, we're agreed. Our new friend is going to be Iron Man. (Episode 9, Season1) 
In order to understand the humor effect we need to know the culture background of the "Iron Man" to appreciate the humor contained in the language meme. So in the sentence "let's see: money, women, technology. Okay, we're agreed. Our new friend is going to be Iron Man", the language meme "Iron Man" produces the humorous effects. Therefore, in order to have a good understanding of the verbal humor in this conversation, we need to know the default context and the new context of the language meme "Iron Man". The production of the verbal humor lies in the disharmony between the default context and the new context of the word "Iron Man". In the conversation, Sheldon, Howard and Leonard are talking about the condition of getting a new friend who should be rich, know a lot of women and interested in technology. And we all know

That Iron Man is a popular American action film and the leading role in the film is called Iron Man, which can't be existed in the real world. To some extent, the features of Iron Man can fit the conditions of the description of friend from Sheldon, Howard and Leonard. So Leonard says "let's see: money, women, technology. Okay, we're agreed. Our new friend is going to be Iron Man." The exchange of the context of the word "Iron Man" generates humorous effects. The default context of the meme "Iron Man" is known to us, but the new context is unknown. And the sudden cognitive transformation of a strained expectation into nothing have generated humorous effects.

Example 4

Leonard: All right, fine. So you want to get back together with her, but you're too ashamed to face her because of whatever it is you did.

Howard: In a nutshell.

Leonard: Okay. Well, how about this? Kidnap Bernadette from the opera wearing a creepy mask so she doesn't know it's you.

Howard: Now, you see, I don't know if you're kidding or not. (Episode 4, Season 4)

The verbal humor lies in the sentence "Kidnap Bernadette from the opera wearing a creepy mask so she doesn't know it's you". The interpretation of verbal humor contained in this sentence requires the default context of the language meme. The culture background is needed. The default context of this sentence is that this is a sentence that comes from The Phantom of the Opera. In the film, it is said that there is a phantom of the opera in the theater, who covers his deformed face with a mask. The phantom of the opera finds that an actress named Christine has a nice voice and he falls in love with her. But Christine falls in love with Raoul, which make the phantom of the opera very angry. He catches Christine into the basement from the theatre with his face covered by a mask. In the above conversation, Leonard is giving Howard the suggestion about how to restore the relationship with Howard's girlfriend. In this new context, Leonard says "Kidnap Bernadette from the opera wearing a creepy mask so she doesn't know it's you", which is actually used to ridicule Leonard. The disharmony between the default context and the new context leads to the humorous effect. The default context of "Kidnap Bernadette from the opera wearing a creepy mask so she doesn't know it's you" is known and the new context is unknown. Transforming from the default context to the new context is the process of leaping from something known to something unknown which can generate the humorous effects by turning the hearer's cognitive transformation of expectation into nothing.

\section{The Process of Humor Generation}

According to Francis Heylighen (1998), the process of meme replication and transmission consists of four stages: assimilation, retention, expression, transmission. This thesis holds the opinion that the process of the generation of verbal humor that is based on language humor also includes the same four stages.

The previous examples from The Bigbang Theory will be used to present the process of the generation of verbal humor.

Example 5

Leonard: If we do get a new friend, he should be a guy you can trust. You know, a guy who has your back.

Howard: And he should have a lot of money and live in a cool place down by the beach where we could throw parties.

Sheldon: And he should share our love of technology.

Howard: And he should know a lot of women.

Leonard: Okay, let's see: money, women, technology. Okay, we're agreed. Our new friend is going to be Iron Man. (Episode 9, Season 1)

The first stage of generation of the language meme "Iron Man" is assimilation. In order to be a successful meme, 
the meme "Iron Man" have to infect a new host and stay in his mind for as long as it can. Leonard is the new host that is infected by the meme. He has met the meme "Iron Man" in the film Iron Man. A meme has to be outstanding in order to be assimilated. Then the meme is noticed, understood and accepted by the new infector Leonard. Leonard can notice it because the meme "Iron Man" is very outstanding and special. After noticing the meme, the host may take the meme into his cognitive system, that is, to understand it. The meme is present in the host's mind deeply and the host is willing to believe it or treat it seriously. If the meme "Iron Man" failed to be assimilated by the host, then no humorous effect can be generated. Therefore, the assimilation is the first and also the preparation stage.

The second stage of generation of verbal humor is the retention of the meme "Iron Man". If the meme "Iron Man" wants to be created, it has to remain in the mind of the host for long enough. There are many other memes, at the same time, existing in the host's mind. The memes will compete with each other to get survived. Through strong selection, only a few memes can survive. So the longer the meme "Iron Man" stays in the mind, the more possibility it will get to be transmitted. And the retention of the meme "Iron Man" in the mind of the host not only relies on the importance and specialty of the meme but also relies on how often it gets repeated. The meme "Iron Man" is from the famous film Iron Man, which enjoys the popularity and is known almost by everyone. So it will be mentioned from time to time, giving it more chance to stay in Leonard's mind and finally get used in conversation which generates humorous effect.

The third stage of generating verbal humor is to express the meme "Iron Man" stored in the mind. In order to be perceived and used in communication, the meme must be transformed into a physical shape from the memory pattern. And speech is most basic medium to express. When Leonard communicates with friends about getting a new friend, the conditions of the new friend remind him of the meme "Iron Man". Leonard thinks that it is appropriate to express it in that context. Thus the expression leads to the disharmony between forms and contents of the meme "Iron Man", generating the humorous effect. This is the generating stage of verbal humor which means that expression is a very important stage for the generation of verbal humor. Speech is a commonly used medium of expression. Beside speech, pictures, texts, behaviors and so on are all the means for expression.

The last stage is the transmission of the meme "Iron Man". Since the humorous effect has already been generated, this stage is more about appreciation of verbal humor. Once the humorous effect is formed, it will transmitted to more individuals by different medias such as speech, pictures, texts, behaviors and so on. The humorous expression may be replicated and transmitted from host to host. Thus the verbal humor generated by Leonard and his friends in the Bigbang Theory like "let's see: money, women, technology. Okay, we're agreed. Our new friend is going to be Iron Man" are transmitted from one to another. So the transmission of verbal humor can be regarded as the appreciating stage.

\section{Conclusion}

The article focuses on the explanation of the process of the generation of verbal humor. This thesis firstly introduced the definition, classification and features of humor. Secondly, the generation of verbal humor, the process of generating verbal humor is elaborated. The generation of verbal humor can be explained from the aspects of meme variation and the disharmony between default context and new context. Thirdly the process of meme replication and transmission consists of four stages: assimilation, retention, expression, transmission. It has some implications.

First, theoretically, through the study on how verbal humors contain language memes are generated and how humorous effect is produced based from memetics, a systematical study of memetics is more and clear. Readers will possess a better understanding of memetic theory and its relevant studies, such as how it is studied, how it is developed, the applications of memetic theory, how it can be used in other fields. First and foremost, readers can comprehend the relationship between memetic theory and verbal humors. Through the interpretation of how humorous effect is produced from the perspective of memetics, readers will have a good skill in interpreting and understanding verbal humors from other foreign counties. The thesis also has significant practical importance to English learning and teaching. As we have known the generation process of verbal humor, it will become much easier for us to appreciate verbal humor in English sitcoms which is beneficial for us to improve English.

\section{Acknowledgment}

This research is supported by Scientific Research Fund Project of Inner Mongolia University for Nationalities, NO. NMDYB15045. 


\section{References}

Blackmore, S. (1999). The Meme Machine (p. 66). Oxford: Oxford University Press.

Blackmore, S. (2001). Evolution and Memes: The Human Brain as a Selective Imitation Device. Cybernetics and Systems, 32(32), 225-255. https://doi.org/10.1080/019697201300001867

Chesterman, A. (1997). Memes of Translation. Amsterdam: John Benjamins. https://doi.org/10.1075/btl.22

Dawkins, R. (1976). The Selfish Gene (p. 206). New York: Oxford University Press.

Deacon, T. (1997). The Symbolic Species: The Coevolution of Language and the Human Brain. London: Penguin.

He, Z. R. (2005). Language Meme, 6, 55. Language Science.

He, Z. R. (2009). New Introduction to Pragmatics (p. 341). Beijing: Beijing University Press.

Heylighten, F. (1998). What Makes a Meme Successful: Selection Criteria for Cultural Evolution (p. 27). Paper presented at 15 th International Congress on Cybernetics.

Koestler, A. (1964). The Act of Creation. London: Hutchinson \& Co.

Lin, Y. T. (2000). My Country and My People. Beijing: Foreign Language Teaching and Research Press.

Lynch, A. (1996). Though Contagion: How Belief Spreads through Society (p. 93). New York: Basic Books.

Xu, L. X. (2003). Discourse Analysis of Humor. Kaifeng: He Hanan University Press.

\section{Copyrights}

Copyright for this article is retained by the author(s), with first publication rights granted to the journal.

This is an open-access article distributed under the terms and conditions of the Creative Commons Attribution license (http://creativecommons.org/licenses/by/4.0/). 